\title{
Enhancing solar cell efficiency by lenses on the nano- and microscale
}

\author{
M. Schmid*a,b P. Manley ${ }^{\mathrm{a}}$ \\ ${ }^{a}$ Helmholtz-Zentrum Berlin, Nanooptical Concepts for PV, Hahn-Meitner-Platz 1, 14109 Berlin, \\ Germany; ${ }^{b}$ Freie Universität Berlin, Department of Physics, Arnimallee 14, 14195 Berlin, Germany
}

\begin{abstract}
Metallic nanoparticles exhibiting plasmonic effects as well as dielectric nanoparticles coupling the light into resonant modes have both shown successful application to photovoltaics. On the larger scale, microconcentrator optics promise to enhance solar cell efficiency and reduce material consumption. Here we want to make the link between concentrators on the nano- and on the microscale. From metallic nanospheres we turn to dielectric ones and then look at increasing radii to approach concentrator optics on the mircoscale. The nano- and microlenses are investigated with respect to their interaction with light using 3D simulations with the finite element method. Resulting maps of local electric field distributions reveal the focusing behavior of the dielectric spheres. For larger lens sizes, ray tracing calculations can be applied which give ray distributions in agreement with areas of high electric field intensities. Calculations of back focal lengths using ray tracing coincide with results from geometrical optics simulations. They give us insight into how the focal length can be tuned as a function of particle size, but also depending on the substrate refractive index and the shape of the microlens. Turning from spherical to segment-shaped lenses allows us to approach the realistic case of microconcentrator optics and to draw conclusions about focus tuning and system design. Despite the similarities of focusing behavior we find for the nano- and the microlenses, the integration into solar cells needs to be carefully adjusted, depending on the ambition of material saving, concentration level, focal distance and lens size, all being closely related.
\end{abstract}

Keywords: light coupling, plasmonics, nanosphere, microlens, concentration, focal length, ray tracing, solar cell

\section{INTRODUCTION}

Plasmonic absorption enhancement by metal nanoparticles has been identified as a promising concept for boosting solar cell efficiency [1]. High angle scattering, enhanced near-fields and in the case of regular particle arrangements the coupling into guided modes are the main mechanisms identified to improve light absorption [2]. As a result, plasmonic nanoparticles have been studied for various types of solar cells, like silicon [3], organic [4], dye-sensitized, GaAs and recently also chalcopyrite-based ones [5]. Another approach for light trapping by nanoparticles which comes with the search for efficiency, but also easy and stable integration into the solar cell are dielectric nanoparticles. Related effects of light coupling can be attributed to whispering gallery modes [6] but also general anti-reflection effects play a fundamental role [7]. Compared to their metallic counterparts, dielectric nanoparticles may show equally strong scattering behavior, yet without any related absorption losses. They may however lose in terms of high angle scattering, yet exhibit a strong forwards scattering [8]. Firstly, we will briefly revisit near-fields around metallic and dielectric nanospheres and then turn to dielectric spheres of increasing size, which show a progressive trend towards a defined focal spot.

Micrometer-sized dielectric spheres have been identified to give rise to so called photonic nanojets, which were early identified to have the potential for a novel ultramicroscopy technique [9]. Detailed studies of the nanojet evolution as a function of parameters like wavelength, particle size and refractive index of sphere and surrounding have followed [10, 11]. In this paper we want to focus on the similarity of light concentration behind dielectric particles and the well-known focusing behavior of lenses. We will show how the focus of the nanojets can be equally described by ray tracing methods as well as in geometrical optics calculations for light propagation through a lens, for the case of microspheres large compared to the wavelength. The transition to plano-convex lenses will be highlighted since this relates to the particular application in solar cells which has recently attracted attention: microconcentrator solar cells. In this concept the sunlight is concentrated by micro-optics onto micrometer-sized solar cells for the purpose of material saving and efficiency enhancement $[12,13]$. By establishing the link between the nano-scaled metallic and dielectric particle concentrators on the one hand and the lens-like micro-concentrators on the other hand we aim at providing a unified picture of light concentration for efficiency enhancement particularly in photovoltaic devices.

*martina.schmid@helmholtz-berlin.de; phone 4930 8062-43243; www.helmholtz-berlin.de 


\section{APPROACH AND COMPUTATIONAL METHODS}

The modification of light propagation by nano- and micrometer-sized spheres, hemispheres and plano-convex lenses is investigated. We start with a comparison of small metallic $(\mathrm{Ag})$ versus dielectric $\left(\mathrm{SiO}_{2}\right)$ nanospheres and then focus on the dielectric case when investigating increasing sizes and various shapes. Ag refractive index data is interpolated to $0.14221+4.52296 i$ according to data from [14], whereas the refractive index for $\mathrm{SiO}_{2}$ is set to $n=1.53839$ (according to [15]) for the wavelength $\lambda=700 \mathrm{~nm}$ investigated here. We focus on this wavelength due to its significance for the application to Chalcopyrite solar cells, which require an absorption enhancement in the range centered on this wavelength. Chalcopyrites, or $\mathrm{Cu}(\mathrm{In}, \mathrm{Ga}) \mathrm{Se}_{2}$ (CIGSe) compounds, are used as absorber materials in thin-film solar cells due to their direct band gap and resulting good efficiencies. But they also come with the aim of reducing material consumption since they contain the rare element indium. When thinking about the application of concentrator lenses to this type of solar cell the refractive index of the absorber layer which is approximately 2.8 and the one of $\mathrm{ZnO}$, used as a front contact for the solar cell, which is approximately 1.8 are of interest. We will use them exemplarily when assigning particular refractive indices to substrates later on. The imaginary part of the refractive index of the substrate will be kept to zero in order to follow the light propagation. For investigating the intensity distributions and focusing effects of the nano- and microlenses three approaches are followed: 3D optical simulations using the finite element method, ray tracing and geometrical optics calculations. The finite element method and ray tracing are briefly described in the following, whereas Sec. 3 is dedicated to the geometrical optics calculations. The generalized structure of the shapes can be seen in Fig. 1 as used for the geometrical optics calculations.

3D optical simulations are carried out using the software package JCMwave [16, 17]. It is based on the finite element method (FEM), i.e. the computational domain is divided into geometrical primitives with a discretization adaptable to the particular geometry. Using local polynomial functions the solution space to Maxwell's equations in the timeharmonic ansatz for the entire domain can be approximated. In the examples presented here, the cylindrical symmetry of the geometry has been exploited to reduce the computational effort. This allows the solutions to Maxwell's equations to be expanded in a Fourier basis which accounts for the angular dependence [18]. The computational domain was terminated by PML (perfectly matched layer) boundary conditions. The total electric field surrounding the particle was calculated. Due to the memory requirements of the FEM, a computational domain size which is larger than a few tens of wavelengths becomes costly, even when exploiting the cylindrical symmetry as explained above. This meant that for our chosen wavelength of $700 \mathrm{~nm}$, calculations for particle diameters larger than $10000 \mathrm{~nm}$ became very time consuming using the FEM. According pictures shown in the following represent the distribution of the electric field normalized to the incoming field $|E||| E_{0} \mid$ displayed in the $x z$-plane at $y=0$; the $z$-direction is defined as the negative propagation direction of the incident light with polarization along the $x$-direction.

Sequential ray tracing describes the propagation of light rays when traveling from an object to an image plane via a given sequence of surfaces. At each of the surfaces the ray propagation is described according to the laws of reflection and refraction and no paraxial approximation is made. By following the path of a ray its angle of incidence onto an optical surface can be identified and Snell's law applied accordingly. Ray tracing is mostly used for the design of optical elements and systems and in this sense is very suitable for our investigations of micrometer-sized lenses where near-field effects are negligible. For the calculations shown here we used the software Radiant Zemax, Version 13 [19].

\section{GEOMETRICAL OPTICS CALCULATIONS}

Geometrical optics or ray optics can be used to describe light propagation through media and objects much larger than the wavelength. Based on this approach we want to derive the rear focal length $f$ and the back focal length $B F L$ for a generalized structure of lens as depicted in Fig. 1. The lens is defined by two curved surfaces with radii $r_{1}$ and $r_{2}$ and a total thickness of $d$ and has a refractive index of $n_{2}$ whilst being embedded in an incoming medium with $n_{1}$ and an outgoing medium with $n_{3}$, eventually also on top of a substrate with $n_{4}$. 


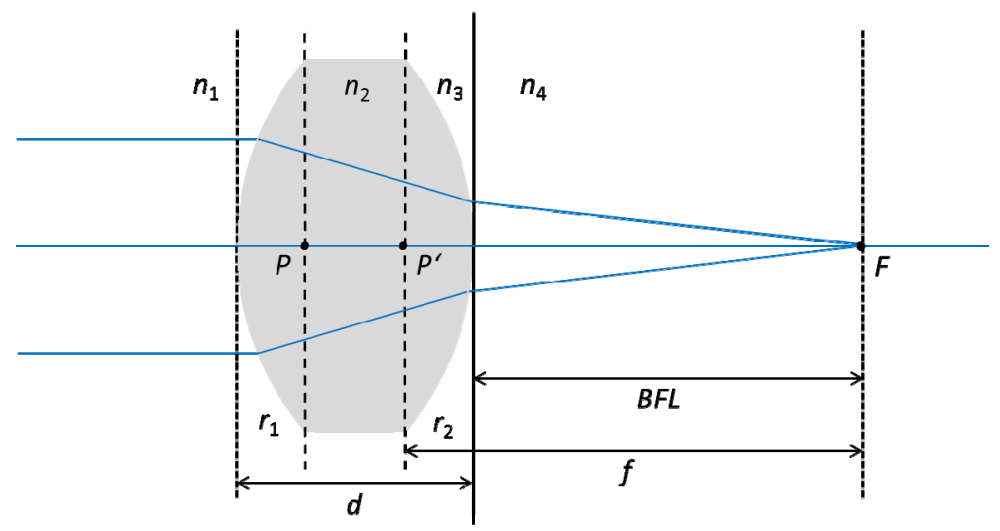

Figure 1. Schematic of a generalized lens structure defined by two curved surfaces with radii $r_{1}$ and $r_{2}$ and a total thickness of $d$ with the lens having a refractive index of $n_{2}$ whilst being embedded in an incoming medium with $n_{1}$ and an outgoing medium with $n_{3}$, eventually also on top of a substrate with $n_{4}$.

The power $\phi$ of an optical surface with radius $r$ between two media with refractive index $n$ and $n$ ' is defined by (see e.g. [20])

$$
\phi=\frac{n^{\prime}-n}{r}
$$

The effective focal length $E F L$ is simply given as $E F L=1 / \phi$, with the front and the rear focal length, however, being expressed by

$$
f_{F}=-\frac{n}{\phi} \quad \text { and } \quad f_{R}=f=\frac{n^{\prime}}{\phi}
$$

respectively. In the Gaussian reduction the total power $\phi$ is combined from the powers $\phi_{1}$ and $\phi_{2}$ of the individual interfaces as

$$
\phi=\phi_{1}+\phi_{2}-\phi_{1} \phi_{2} \frac{d}{n_{2}}
$$

with the center thickness $d$ and the refractive index $n_{2}$ of the intermediate (lens) material. In our particular case of Fig. 1 and considering Eqs. (1) and (2) this leads us to the condition for the (rear) focal length $f_{R}=f$ in a

Generalized Lensmaker's Equation:

$$
\frac{1}{f}=\frac{n_{2}-n_{1}}{n_{4}} \cdot \frac{1}{r_{1}}-\frac{n_{2}-n_{3}}{n_{4}} \cdot \frac{1}{r_{2}}+\frac{\left(n_{2}-n_{1}\right) \cdot\left(n_{2}-n_{3}\right)}{n_{2} \cdot n_{4}} \cdot \frac{d}{r_{1} \cdot r_{2}}
$$

For the derivation of the front focal length $F F L$ and the back focal length $B F L$ we have to consider the primary and the secondary principle point $P$ and $P^{\prime}$, respectively. They are given by

$$
P=\frac{\phi_{2}}{\phi} \frac{n}{n_{2}} d \quad \text { and } \quad P^{\prime}=-\frac{\phi_{1}}{\phi} \frac{n^{\prime}}{n_{2}} d
$$

which together with the relations

$$
F F L=f_{F}+P \quad \text { and } \quad B F L=f_{R}+P^{\prime}
$$

results in the equation for our parameter of interest 


$$
B F L=f \cdot\left(1-\frac{n_{2}-n_{1}}{n_{2}} \cdot \frac{d}{r_{1}}\right)
$$

The particular shapes investigated are spheres, hemispheres and circular segments in air and on a substrate. The spherical elements are described by the radius $r$ and the segments by the segment width $s$ and the height $h$, resulting in $r=\left(4 h^{2}+s^{2}\right) /(8 h)$ for the latter ones. With the definition of the height as a certain ratio this can be expressed as

$$
r_{\text {segment }}=\frac{s \cdot\left(4 \cdot \text { ratio }^{2}+1\right)}{8 \cdot \text { ratio }} \quad \text { for ratio }=h / s
$$

The resulting equations for the rear focal point and the back focal length for the various cases are summarized in Table 1.

Table 1. Formulas for the rear focal point and the back focal length for spheres, hemispheres and segments in air and on a substrate according to the generalized lensmaker's equation, Eq. (4), and to Eq. (7) for the back focal length.

\begin{tabular}{|l|l|l|}
\hline Configuration & Focal length & Back focal length \\
\hline $\begin{array}{l}\text { Sphere of radius } r=r_{1}=-r_{2} \text { with refractive index } n=n_{2} \\
\text { in air }\left(n_{1}=n_{3}=\mathrm{n}_{4}=1\right) ; d=2 r\end{array}$ & $\frac{1}{f}=\frac{n-1}{n} \cdot \frac{2}{r}$ & $B F L=f \cdot \frac{2-n}{n}=\frac{2-n}{2(n-1)} \cdot r$ \\
\hline $\begin{array}{l}\text { Sphere of radius } r \text { with refractive index } n_{2}=n \text { in air }\left(n_{1}\right. \\
\left.=n_{3}=1\right), \text { but on a substrate with } n_{4}=n_{\text {subs }}\end{array}$ & $\frac{1}{f}=\frac{n-1}{n \cdot n_{\text {subs }}} \cdot \frac{2}{r}$ & $B F L=f \cdot \frac{2-n}{n}=\frac{(2-n) \cdot n_{\text {subs }}}{2(n-1)} \cdot r$ \\
\hline $\begin{array}{l}\text { Hemisphere of radius } r=r_{l}=d, r_{2}=\infty \text { with refractive } \\
\text { index } n=n_{2} \text { in air }\left(n_{1}=n_{3}=\mathrm{n}_{4}=1\right)\end{array}$ & $\frac{1}{f}=(n-1) \cdot \frac{1}{r}$ & $B F L=f \cdot \frac{1}{n}=\frac{1}{n(n-1)} \cdot r$ \\
\hline $\begin{array}{l}\text { Hemisphere of radius } r \text { with refractive index } n_{2}=n \text { in } \\
\text { air }\left(n_{1}=n_{3}=1\right), \text { but on a substrate with } n_{4}=n_{\text {subs }}\end{array}$ & $\frac{1}{f}=\frac{n-1}{n_{\text {subs }}} \cdot \frac{1}{r}$ & $B F L=f \cdot \frac{1}{n}=\frac{n_{\text {subs }}}{n(n-1)} \cdot r$ \\
\hline $\begin{array}{l}\text { Segment of width } s, \text { height } h=s \cdot \text { ratio }=d, \text { radius } r \\
\text { according to Eq. (8) and with refractive index } n=n_{2} \text { in } \\
\text { air }\left(n_{1}=n_{3}=\mathrm{n}_{4}=1\right)\end{array}$ & $\frac{1}{f}=(n-1) \cdot \frac{1}{r}$ & $B F L=f \cdot\left(1-\frac{n-1}{n} \cdot \frac{h}{r}\right)$ \\
\hline $\begin{array}{l}\text { Segment of width } s, \text { height } h=s \cdot r a t i o, \text { radius } r \text { and with } \\
\text { refractive index } n_{2}=n \text { in air }\left(n_{1}=n_{3}=1\right), \text { but on a } \\
\text { substrate with } n_{4}=n_{\text {subs }}\end{array}$ & $\frac{1}{f}=\frac{n-1}{n_{\text {subs }}} \cdot \frac{1}{r} \cdot r-\frac{1}{n} \cdot h$ \\
\hline
\end{tabular}

\section{RESULTS AND DISCUSSION}

Looking at spheres on the nanoscale, a strong near-field enhancement is one of the reasons why metallic nanoparticles are of interest for absorption enhancement. Fig. 2a) shows the normalized electric near-field distribution around a $\mathrm{Ag}$ nanoparticle with $200 \mathrm{~nm}$ radius at a wavelength of $700 \mathrm{~nm}$. The representation is a cut through the $x z$-plane at $y=0$ with polarization along the (horizontal) $x$-direction and the $k$-vector along the negative $z$-direction. It clearly reveals a high concentration of field intensity around the boundary of the metallic nanoparticle, which due to the free charge carriers repels fields from its inside. Additionally, the lobes of this quadrupole mode are visible, highlighting the second benefit of plasmonic nanoparticles, namely the reradiation of light into high angles, potentially leading to light trapping in an underlying layer. Despite these benefits, metallic nanoparticles may also show parasitic absorption resulting in losses in the form of heat. This disadvantage together with the challenge of stable integration into solar cells [21] leads to the consideration of nanoparticles made from dielectric materials, which can equally show high scattering ability with similar resonance wavelengths for slightly larger sizes. A detailed comparison of metallic versus dielectric nanoparticles 
was conducted in [8]. Here, we focus on the very particular electric field distribution around dielectric nanoparticles as shown in Fig. 2b) for the example of a $\mathrm{SiO}_{2}$ nanosphere with $r=300 \mathrm{~nm}$. The light is preferentially guided to the forwards direction and concentrated in the shadow-region behind the nanoparticle. This focusing effect already observable for dielectric particles smaller than the wavelength will be a center point of our discussion as we proceed now to larger sphere sizes.

a)

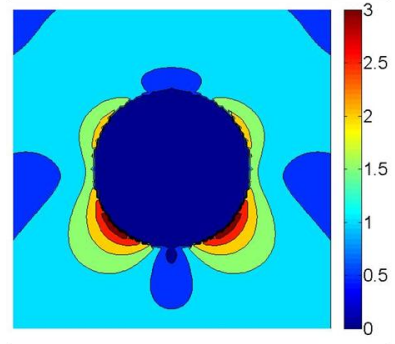

b)

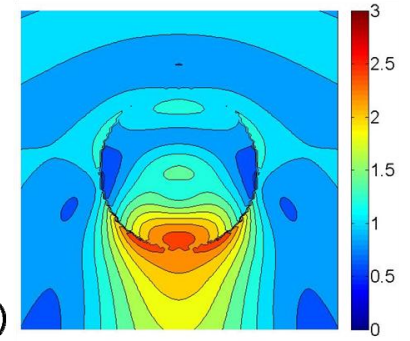

Figure 2. Electric near-field distribution around a metallic versus a dielectric nanoparticle: a) Ag nanoparticle with radius $200 \mathrm{~nm}, \mathrm{~b}$ ) $\mathrm{SiO}_{2}$ nanoparticle with radius $300 \mathrm{~nm}$ in air; depicted is the electric field in the $x z$-plane normalized to the incident field with polarization along the $x$-direction and $k$-vector along the negative $z$-direction at a wavelength of $700 \mathrm{~nm}$.

Fig. 3 reveals how the point of maximum field shifts away from the sphere with increasing radius. Fig. 3a)-c) show the electric field distribution around a $\mathrm{SiO}_{2}$ dielectric sphere with radius of $1 \mu \mathrm{m}, 2 \mu \mathrm{m}$ and $3 \mu \mathrm{m}$, respectively. The maximum of the field, just being on the border of the sphere for the case of $r=1 \mu \mathrm{m}$, gradually moves outwards and approaches the back focal point as calculated from geometrical optics (Tab. 1) and marked by the cross. Fig. 3d) gives the dependency of the point of maximum intensity (PMI) normalized to the respective radius as a function of $r$. For radii smaller than $1 \mu \mathrm{m}$ it is negative which means the PMI is inside the sphere. For increasing radii the PMI increases and starts to saturate. A linear dependency of the back focal point on the radius is expected in geometrical optics and was also calculated in 3D simulations e.g. for microcylinders by [22]. From the $r$-range simulated here, a PMI of maximum 0.19 can be fitted, whereas the geometrical optics calculation gives 0.43 . The saturation appears not to be fully reached yet, but also another fact comes into play, namely that the maximum intensity resulting from calculations of electric field distributions was in our simulations always observed to stay below the value of the geometrical back focal point. We attribute this to the particular intensity distribution in the high intensity region as described by [11]. The profile of optical intensity of the nanojet in the direction of propagation is asymmetric with the maximum at shorter distances. Thus the z-value attributed to the center of the high intensity region deviates from the z-value of the absolute maximum.

a)

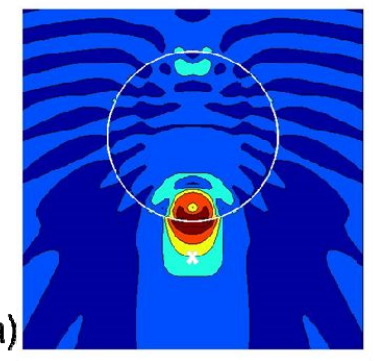

b)

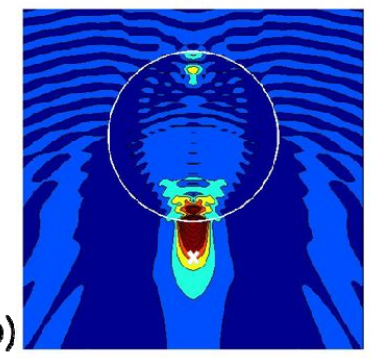

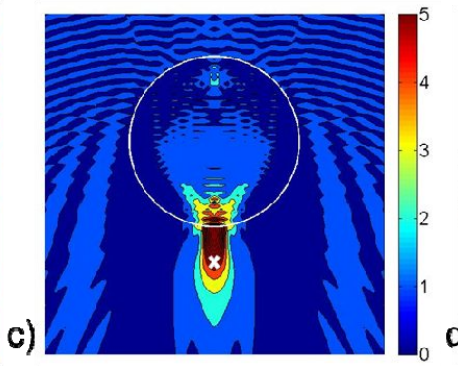

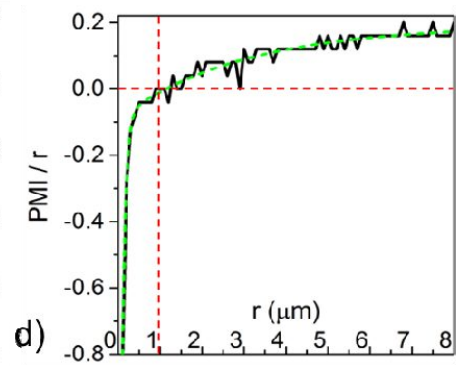

Figure 3. Normalized electric field distribution at $\lambda=700 \mathrm{~nm}$ around a $\mathrm{SiO}_{2}$ nanoparticle with increasing radius $r$ of a) $1 \mu \mathrm{m}$, b) $2 \mu \mathrm{m}$ and c) $3 \mu \mathrm{m}$; the shape of the sphere is marked for clarity as well as the back focal length calculated from geometrical optics (cross); d) gives the point of maximum intensity with respect to the sphere's rear surface normalized to its radius and plotted as a function of the radius.

However, when comparing the light distribution resulting from ray tracing simulations to the electric field distributions extracted from simulations with the 3D FEM, we find a good overall agreement already for spheres with $r=2.5 \mu \mathrm{m}$. Fig. 4a)-c) show a $\mathrm{SiO}_{2}$ sphere in air and on a substrate with constant refractive index $n=1.8$ or $n=2.8$, respectively, which can be attributed to the deposition on top of the front $\mathrm{ZnO}$ contact or on top of the CIGSe absorber when correlating to the application in solar cells. An absorption coefficient of zero was chosen for the substrate layers to fully follow the ray propagation without any absorption losses. The ray propagation is plotted as an overlay to the electric field distribution which reveals that the focus of the rays falls well into the area of highest field enhancement. With increasing substrate 
refractive index the focus shifts away from the sphere, showing us that an adequate choice of the substrate material is one option to tune the PMI / the back focal length.

a)

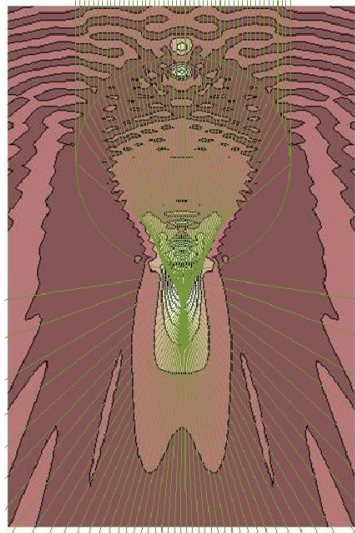

b)

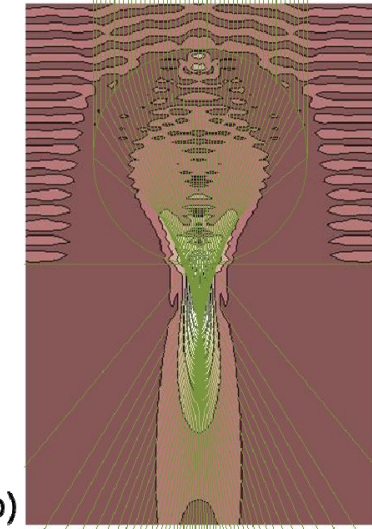

c)

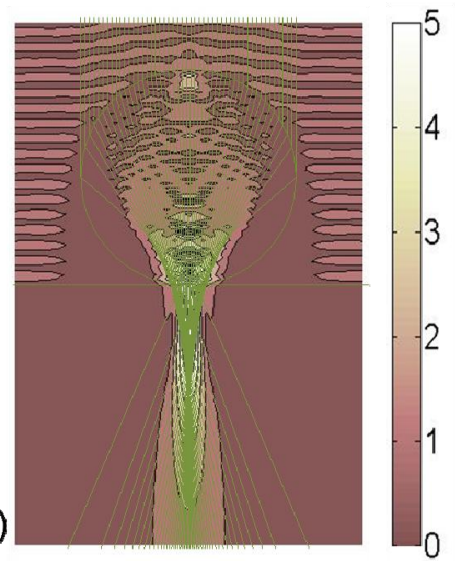

Figure 4. Focus of $\mathrm{a} \mathrm{SiO}_{2}$ sphere with $r=2.5 \mu \mathrm{m}$ a) in air, b) on an $n=1.8$ substrate, c) on an $n=2.8$ substrate, seen in electric field distribution (brown/tan) and ray tracing (green).

Another way of tuning the focal length is by changing the shape of the lens. By moving from a spherical to a hemispherical and from this further to segment-shaped lenses we can also increase the back focal length $B F L$. The diameter $d$ which becomes the segment width $s$ is kept constant while reducing the segment height $h$. This leads to the radius of curvature of the lens increasing as $h$ decreases, which can be seen in Fig. 5. For clarity only ray tracing results are shown. By flattening of a plano-convex lens in this way, the $B F L$ can be increased from $0.60 * d$ for a hemispherical $\mathrm{SiO}_{2}$ lens to $0.86 * s$ for an $h=0.3 *^{*}$ segment and further to $2.53 * s$ for an $h=0.1 *_{s}$ segment.

a)

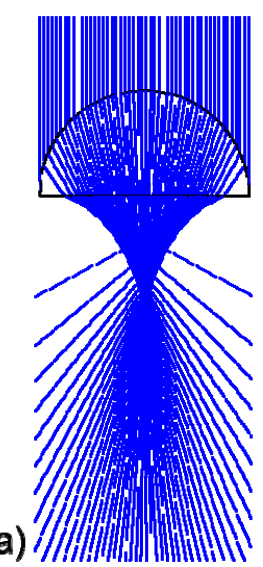

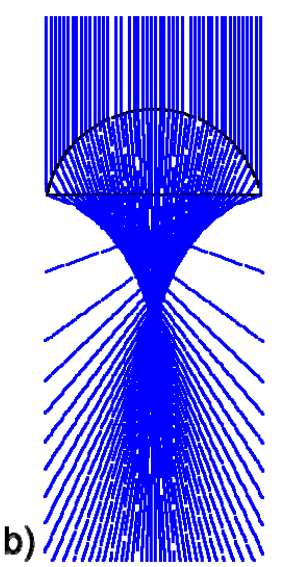

c)

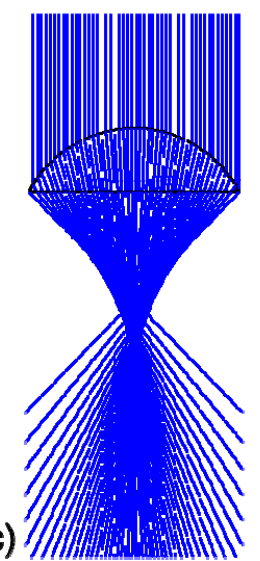

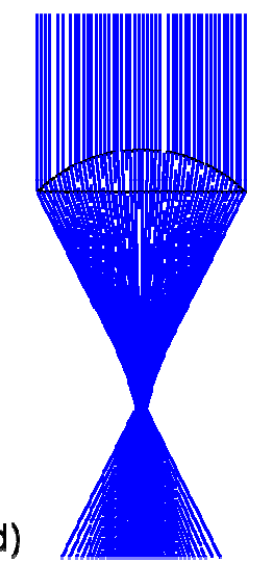

Figure 5. Increasing back focal length of plano-convex lens made from $\mathrm{SiO}_{2}$ with decreasing height and increasing radius of curvature. The height is given as a ratio of the segment width with ratio =a) 0.5 (hemisphere), b) 0.4, c) 0.3 and d) 0.2 .

Fig. 6 summarizes the back focal lengths as calculated by the geometrical optics approach (formulas see Tab. 1) and which agree within deviations of $\leq 0.3 \%$ with the results from ray tracing simulations (Zemax). As given by the respective formula in Tab. 1, the $B F L$ linearly increases with the substrate refractive index, see Fig. 6a) for a $\mathrm{SiO}_{2}$ sphere in air and on a substrate with increasing refractive index $n_{\text {subs }}$. The values for the cases represented in Fig. 4 are marked with a cross. According to the formula in Tab. 1 and considering Eq. (8) for segment-shaped lenses the $B F L$ is even more sensitive to the shape, i.e. here changes in the relative height $h$ represented by the variable ratio $=h / s$, where $s$ is the segment width. Fig. 6b) shows the $B F L$ as a function of $\mathrm{s} / h$ for $\mathrm{SiO}_{2}$ segments in air which reveals an almost linear dependency, especially for larger values (smaller heights). This means, the $1 / h$ term is dominating, making the changes of relative height to an approach much more sensitive for adaptation of the $B F L$ compared to variations of substrate refractive index where the $B F L$ only goes linearly with $n_{\text {subs }}$. Comparing the results presented here, we can conclude that both substrate refractive index and lens shape can have a marked impact on the BFL. However as Fig. 6 shows, the 
shaping of the lens offers more scope for change in the value of $B F L$, especially when considering that the substrate composition may need to be optimized for electronic rather than optical efficiency.
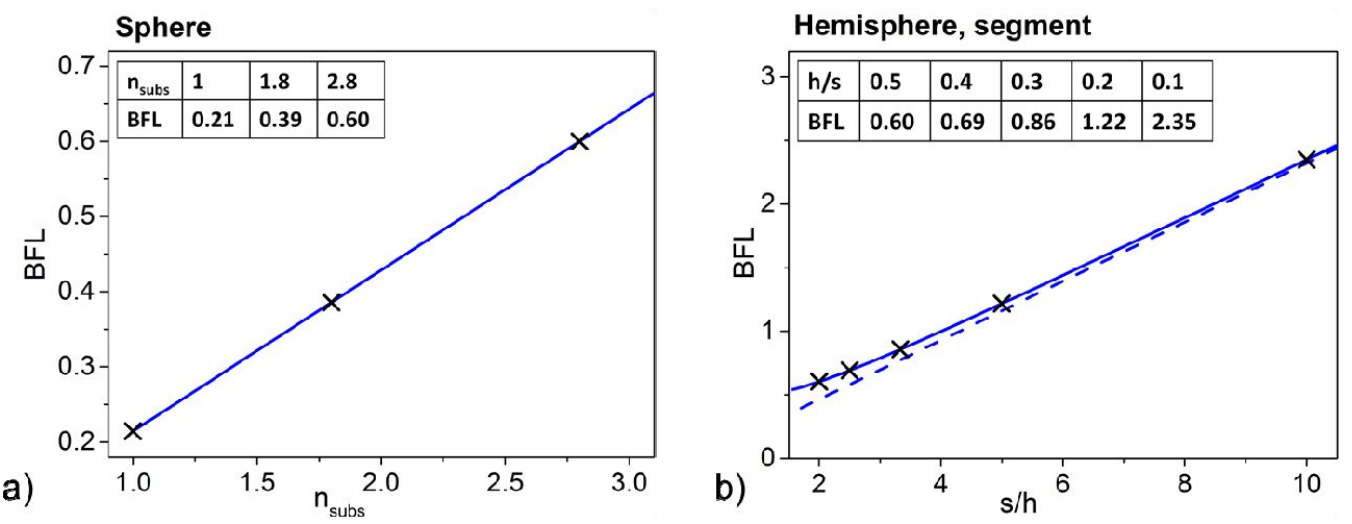

Figure 6. Back focal length (according to Tab. 1 for geometrical optics calculations which agree with results from ray tracing simulations) for a) a $\mathrm{SiO}_{2}$ sphere in air and on a substrate and b) $\mathrm{SiO}_{2}$ hemispheres and segments in air; the total curve progressions are shown with marks for the particular cases represented in Fig. 4 and 5 ( $s$ or $d$ set to 1).

\section{OUTLOOK}

Both increased substrate refractive index and reduced lens height essentially shift the focal point further from the lens. This may not be desired for our application to solar cells where we are aiming for compact devices. Thus, for this application spherical microlenses appear initially to be more suitable. When a spacer layer of low refractive index is introduced, other lens geometries may be preferable. Looking at the influence of overall lens size, even for a substrate index of $n=1.5$, i.e. glass, and a spherical lens made from $\mathrm{SiO}_{2}$ the back focal length in the case of a $500 \mu \mathrm{m}$ diameter lens is $B F L=320 \mu \mathrm{m}$. For a five-hundred times smaller $\mathrm{SiO}_{2}$ spherical lens with $d=1 \mu \mathrm{m}$ the back focal length in an $n=$ 1.5 substrate layer was only $B F L=0.64 \mu \mathrm{m}$. Therefore, we can distinguish between two aims to be followed by applying nano- and mircolenses to solar cells:

a) vertical material saving, i.e. reduction of absorber layer thickness

with compensation of absorption losses by application of nanolenses leading to enhanced incoupling of light;

lenses characterized by low concentration, short focal lengths and negligible spacing required

b) horizontal material saving, i.e. significant reduction of area covered by absorber material;

full use of the incident light by concentration onto the restricted absorber areas

using microlenses with high concentration but long focal lengths and thus spacer layers required

In the end, detailed investigations of the local electric field distribution will need to be taken into account in order to find the most promising configurations. In the case of microconcentrator solar cells, the question arises of what will be the optimum size of the micro solar cells. An optimum trade-off between a stable device with large material saving and collection of almost all of the incident light must be found. Also the optimum position for a micro solar cell of given size needs to be determined for which the local distributions of electric field intensity can be supportive.

\section{CONCLUSION}

From metallic nanospheres we have made the link to lossless dielectric ones and taken a detailed look at their focusing behavior particularly as a function of size. 3D optical simulations using the finite element method reveal the strong local field enhancement in the shadow region behind the dielectric sphere and highlight the transition from near- to far-field focusing. The quantitative maps of electric field strengths give insight in the local distribution of intensities including the area of maximum light concentration. This focusing area coincides for micrometer-sized spheres with results from ray 
tracing which give the value of the back focal length itself. The results from ray tracing are in agreement with focal lengths calculated according to geometrical optics and give us indications of how the focal length can be tuned apart from adjusting the particle size. Both, an increased substrate refractive index as well as the transition from a spherical to a hemispherical or segment-like shape, can increase the focal length. For the particular application to solar cells with the aim of a compact device design, a short distance of the point of highest intensity from the concentrating lens may be required. This brings us back to spherically-shaped particles of smaller sizes, which may win in terms of field enhancement in the close vicinity of the lens and thus improve devices with very thin absorber layers. With respect to substantial material saving by horizontal reduction of the absorber size, microconcentrator solar cells, however, may be beneficial. They rely on microscaled lenses and can be promising when integrating adequate spacer layers in order to bring the micro cells into the region of high field intensities.

\section{ACKNOWLEDGMENT}

The authors are grateful to the Helmholtz Association for support from the Initiative and Networking fund for the Young Investigator group VH-NG-928.

\section{REFERENCES}

[1] K. R. Catchpole, and A. Polman, "Plasmonic solar cells," Optics Express, 16(26), 21793-21800 (2008).

[2] H. A. Atwater, and A. Polman, "Plasmonics for improved photovoltaic devices," Nature Materials, 9, 205-213 (2010).

[3] S. Pillai, K. R. Catchpole, T. Trupke et al., "Surface plasmon enhanced silicon solar cells," Journal of Applied Physics, 101(9), 093105/1-093105/8 (2007).

[4] L. Fang, Q. Di, X. Qi et al., "Efficiency enhancement in organic solar cells with extended resonance spectrum of localized surface plasmon," IEEE Photonics Journal, 5(4), 8400307/1-8400307/7 (2013).

[5] M. Schmid, R. Klenk, M. C. Lux-Steiner et al., "Modeling plasmonic scattering combined with thin-film optics," Nanotechnology, 22(2), 025204/1-025204/10 (2011).

[6] J. Grandidier, D. M. Callahan, J. N. Munday et al., "Light absorption enhancement in thin-film solar cells using whispering gallery modes in dielectric nanospheres," Advanced Materials, 23(10), 1272-1276 (2011).

[7] C. R. Simovski, A. S. Shalin, P. M. Voroshilov et al., "Photovoltaic absorption enhancement in thin-film solar cells by non-resonant beam collimation by submicron dielectric particles," Journal of Applied Physics, 114(10), 103104 (2013).

[8] M. Schmid, P. Andrae, and P. Manley, "Plasmonic and photonic scattering and near fields of nanoparticles," Nanoscale Research Letters, 9, 50/1-50/9 (2014).

[9] Z. C. a. A. Taflove, "Photonic nanojet enhancement of backscattering of light by nanoparticles: a potential novel visible-light ultramicroscopy technique," Optics Express, 12(7), 1214-1220 (2004).

[10] L. L. a. Z. W. Seoungjun Lee, "Optical resonances in microsphere photonic nanojets,” Journal of Optics, 16(1), (2014).

[11] Y. E. Geints, E. K. Panina, and A. A. Zemlyanov, "Control over parameters of photonic nanojets of dielectric microspheres," Optics Communications, 283(23), 4775-4781 (2010).

[12] J. Yoon, A. J. Baca, S. I. Park et al., "Ultrathin silicon solar microcells for semitransparent, mechanically flexible and microconcentrator module designs," Nature Materials, 7(11), 907-915 (2008).

[13] M. Paire, L. Lombez, J.-F. o. Guillemoles et al., "Toward microscale $\mathrm{Cu}(\mathrm{In}, \mathrm{Ga}) \mathrm{Se}[\mathrm{sub}$ 2] solar cells for efficient conversion and optimized material usage: Theoretical evaluation," Journal of Applied Physics, 108(3), 034907/1-034907/7 (2010).

[14] E. D. Palik, [Handbook of optical constants of solids] Academic Press, Orlando (1985).

[15] [Handbook of Optics, Third Edition Volume IV: Optical Properties of Materials, Nonlinear Optics, Quantum Optics (set)] McGraw-Hill Education, (2009).

[16] J. Hoffmann, C. Hafner, P. Leidenberger et al., "Comparison of electromagnetic field solvers for the 3D analysis of plasmonic nano antennas," Proceedings of SPIE, 7390, 73900J/1-73900J/11 (2009).

[17] www.jcmwave.com. 
[18] S. Burger, B. H. Kleemann, L. Zschiedrich et al., "Finite-element simulations of light propagation through circular subwavelength apertures," Proceedings of SPIE, 7366, 736621/1-736621/8 (2009).

[19] www.zemax.com

[20] J. E. Greivenkamp, [Field Guide to Geometrical Optics] SPIE Press, (2004).

[21] M. Schmid, J. Klaer, R. Klenk et al., "Stability of plasmonic metal nanoparticles integrated in the back contact of ultra-thin $\mathrm{Cu}(\mathrm{In}, \mathrm{Ga}) \mathrm{S} 2$ solar cells," Thin Solid Films, 527, 308-313 (2013).

[22] C.-Y. Liu, "Ultra-high transmission of photonic nanojet induced modes in chains of core-shell microcylinders," Physics Letters A, 3(5), (2012). 